\title{
Long-Range Photon Fluctuations Enhance Photon-Mediated Electron Pairing and Superconductivity
}

\author{
Ahana Chakraborty๑* and Francesco Piazza ${ }^{\dagger}$ \\ Max Planck Institute for the Physics of Complex Systems, \\ Nöthnitzerstrasse 38, 01187 Dresden, Germany
}

(Received 20 August 2020; revised 25 January 2021; accepted 27 September 2021; published 22 October 2021)

\begin{abstract}
Recently, the possibility of inducing superconductivity for electrons in two-dimensional materials has been proposed via cavity-mediated pairing. The cavity-mediated electron-electron interactions are long range, which has two main effects: firstly, within the standard BCS-type pairing mediated by adiabatic photons, the superconducting critical temperature depends polynomially on the coupling strength, instead of the exponential dependence characterizing the phonon-mediated pairing; secondly, as we show here, the effect of photon fluctuations is significantly enhanced. These mediate novel non-BCS-type pairing processes, via nonadiabatic photons, which are not sensitive to the electron occupation but rather to the electron dispersion and lifetime at the Fermi surface. Therefore, while the leading temperature dependence of BCS pairing comes from the smoothening of the Fermi-Dirac distribution, the temperature dependence of the fluctuation-induced pairing comes from the electron lifetime. For realistic parameters, also including cavity loss, this results in a critical temperature which can be more than 1 order of magnitude larger than the BCS prediction. Moreover, a finite average number of photons (as can be achieved by incoherently pumping the cavity) adds to the fluctuations and leads to a further enhancement of the critical temperature.
\end{abstract}

DOI: 10.1103/PhysRevLett.127.177002

Introduction.-The development of experimental solidstate platforms coupling electrons with the quantum light of optical cavities [1,2] offers exciting prospects for the exploration of novel types of collective phenomena, which can arise due to the peculiar nature of the cavity-mediated interactions and their interplay with electronic correlations. Several scenarios have been theoretically investigated, with relevance for solid-state materials [3-11] and ultracold fermionic atoms [12-22].

One particular direction which is receiving considerable attention is the possibility to induce electronic superconductivity via photon-mediated pairing [7,9]. The critical temperature has been predicted to follow a nonexponential dependence on the light-matter coupling strength, which can be explained within the usual BCS paradigm as being due to the long-range character of the cavity-mediated interactions, i.e., the fact that the photons transfer a welldefined momentum.

In this Letter, we show that the long-range nature of the cavity-mediated interactions can have an even more

Published by the American Physical Society under the terms of the Creative Commons Attribution 4.0 International license. Further distribution of this work must maintain attribution to the author(s) and the published article's title, journal citation, and DOI. Open access publication funded by the Max Planck Society. dramatic influence on superconductivity, which is to introduce a novel, non-BCS-type of pairing mediated by on shell, nonadiabatic photon fluctuations. This is different from BCS pairing, which involves instead the emissionabsorption of adiabatic, off shell photons, and depends on the thermal occupation of electrons. On the other hand, the fluctuation-mediated pairing is only directly affected by the electron dispersion and lifetime near the Fermi surface. Therefore, temperature hinders this non-BCS-type of pairing by decreasing the electron lifetime, while it affects the BCS pairing mainly by smoothening the Fermi-Dirac distribution.

This new fluctuation-mediated pairing can enhance superconductivity significantly. Considering a Fermiliquid-type electron lifetime set by the screened Coulomb interaction, the critical temperature can be more than 1 order of magnitude larger than the BCS prediction, using realistic parameters for the terahertz cavities considered in Ref. [9] and including photon loss.

At the typical transition temperatures, optical cavities are unoccupied on average, such that only vacuum fluctuations or loss-induced noise contribute to the non-BCS-type pairing. A finite average number of photons can be achieved by pumping the cavity, which then further amplifies this type of pairing. In this way, the critical temperature can be enhanced at least until the Fermi-liquid picture holds, approaching thus the Fermi temperature 
(slightly above $10 \mathrm{~K}$ for the materials considered in Ref. [9]), even at moderate electron-photon coupling.

Model.-We consider a 2D electron system coupled to a terahertz cavity. The phenomenology discussed here relies on two properties of the cavity field. The first and most important feature is that the cavity-mediated interaction is long ranged. We model this by restricting the momentum transferred by the photon to a fixed vector $\vec{q}_{0}$. This simplest model corresponds to the case of a split-ring cavity [9], sustaining a standing-wave mode of frequency $\omega_{c}$, and momentum $\pm q_{0} \hat{x}$, with $q_{0}=\omega_{c} \sqrt{\epsilon_{r}} / c$. Here, $\epsilon_{r}$ is the relative permittivity of the $2 \mathrm{D}$ material and $c$ is the speed of light in vacuum. We shall show that our results remain essentially unchanged in the limit $q_{0} \rightarrow 0$ as well as by allowing for a broadening of the photon momentum, provided the corresponding frequency width is smaller than the electron lifetime at the Fermi surface (FS). This extends the applicability of our results to the case of the planar microcavity geometry considered in Ref. [7], where the set of propagating transverse modes is distributed around $q=0$ with a narrow width set by $\omega_{c} / c$, where now $\omega_{c}$ is the frequency of the purely longitudinal mode. The second feature we consider here is that the cavity field couples to the electron density. As shown in Ref. [9], this can be achieved by driving the system with a transverse laser beam of frequency $\omega_{L}$ which is detuned from the cavity frequency by $\delta_{c}=\omega_{c}-\omega_{L}$. For sufficiently strong laser driving, the dominant light-matter interaction is induced via two-photon diamagnetic processes [9] and reads

$$
H_{\text {light-matter }}=\sum_{\vec{k}, \sigma} \sum_{\vec{q}= \pm q_{0} \hat{x}} g_{0} c_{\vec{k}+\vec{q}, \sigma}^{\dagger} c_{\vec{k}, \sigma}\left(b+b^{\dagger}\right),
$$

where $b^{\dagger}$ and $c_{\vec{k}, \sigma}^{\dagger}$ are the creation operator of cavity photons of momentum $\vec{q}_{0}$ and electrons of momentum $\vec{k}$ and spin $\sigma$ respectively. The coupling strength $g_{0}$ is tuneable by the intensity of the external laser beam.

Causal structure of the pairing.-We compute the superconducting critical temperature $T_{c}$ via the pairing instability of the vertex function $\Gamma$ in the Cooper pairing channel, involving electrons moving with opposite momenta $\vec{p}$ and $-\vec{p}$. The Dyson equation for the vertex function is known as the Bethe-Salpeter (BS) equation [23], which we formulate using real-time Green's functions (GFs) defined on the Keldysh closed time contour. Besides being suited to include cavity loss (and incoherent pumping), this approach also allows one to clearly separate the non-BCS-type, fluctuation-induced pairing we propose here from the standard BCS pairing. The BS equation in its simplest form has the following structure [see Fig. 1(a) and Appendix B in the Supplemental Material [24] ]:

$$
\Gamma=\Gamma_{0}+\Gamma_{\mathrm{BCS}}+\Gamma_{\text {fluct }}
$$

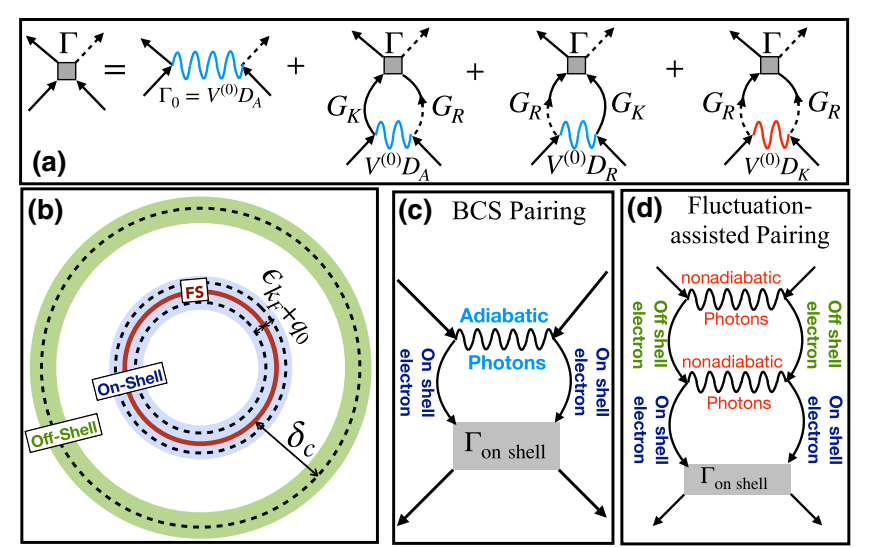

FIG. 1. (a) The real-time Keldysh formulation of the BetheSalpeter equation for the vertex function $\Gamma$ in the pairing channel. It contains the bare vertex $\Gamma_{0}$ (first diagram), the standard BCS terms $\Gamma_{\mathrm{BCS}}$ (second and third diagrams), and the "fluctuation term," $\Gamma_{\text {fluct }}$ (fourth diagram). The latter leads to a non-BCS-type of pairing. (b) The electronic energy structure can be divided between on shell electrons in the vicinity of the FS: $\omega_{1} \sim \epsilon_{k_{F} \pm q_{0}}$ and off shell electrons excited to photonic frequencies: $\omega_{1} \sim \delta_{c}$ [see Eq. (3)]. (c) Standard BCS pairing: the scattering via off shell adiabatic photons leaves the electrons on shell. (d) Fluctuationinduced pairing: on shell nonadiabatic photons at frequencies $\pm \delta_{c}$ induce pairing between on shell electrons through an intermediate transition to off shell electronic states.

where $\Gamma_{0}=V^{(0)} D_{A}$ is the bare vertex. Here $\Gamma(\vec{p}, \omega)$ is a function of the relative momentum and frequency of the incoming electrons. Within the ladder approximation $[23,28]$ for the vertex function, we have

$$
\begin{aligned}
& \Gamma_{\mathrm{BCS}}^{A(R)}(p)=\mathbf{i} \int_{k} V^{(0)} D_{A(R)}(k-p) G_{K(R)}(k) G_{R(K)}(-k) \Gamma(k) \\
& \Gamma_{\text {fluct }}(p)=\mathbf{i} \int_{k} V^{(0)} D_{K}(k-p) G_{R}(k) G_{R}(-k) \Gamma(k),
\end{aligned}
$$

with the coupling function $V^{(0)}(\vec{k}-\vec{p})=g_{0}^{2} \delta_{c} \delta_{\vec{k}-\vec{p}, \pm q_{0} \hat{x}}$. Here, $p=(\vec{p}, \omega), k=\left(\vec{k}, \omega_{1}\right)$ and $\int_{k}=\int d \vec{k} d \omega_{1} /(2 \pi)^{3}$. We denote here the electron and photon GFs by $G$ and $D$, respectively. Within the real-frequency Keldysh formulation, each GF can be of two types: a retarded (advanced) GF [denoted by the subscript $R(A)$ ] or a Keldysh GF (denoted by the subscript $K$ ).

Formulated in momentum-frequency space, the retarded GF contains only information about the dispersion and lifetime of the (quasi)particles, while the Keldysh component explicitly depends also on the occupation of the quasiparticle modes. In thermal equilibrium, these two Green's functions are connected by a fluctuationdissipation relation [29]. We first consider a situation where the whole system is at temperature $T$, and the GFs entering the BS equation read [29] (see Appendix A in the Supplemental Material [24]), 


$$
\begin{aligned}
G_{R(A)}(\vec{k}, \omega) & =\frac{1}{\omega-\epsilon_{k} \pm \mathbf{i} 0^{+}}, \\
G_{K}(\vec{k}, \omega) & =-2 \pi \mathbf{i} \tanh \left(\frac{\omega}{2 T}\right) \delta\left(\omega-\epsilon_{k}\right), \\
D_{R(A)}(\omega) & =\frac{1}{2} \frac{1}{\left(\omega \pm \mathbf{i} 0^{+}\right)^{2}-\delta_{c}^{2}}, \\
D_{K}(\omega) & =\frac{-\pi \mathbf{i}}{2 \delta_{c}}\left[\delta\left(\omega-\delta_{c}\right)-\delta\left(\omega+\delta_{c}\right)\right] \operatorname{coth}\left(\frac{\omega}{2 T}\right) .
\end{aligned}
$$

Here, $\epsilon_{k}$ is the dispersion of the electrons measured from the Fermi energy $E_{F}$. Let us now discuss the physical interpretation of the terms contributing to the BS equation [Eq. (2)]. The standard BCS term $\Gamma_{\mathrm{BCS}}=\Gamma_{\mathrm{BCS}}^{A}+\Gamma_{\mathrm{BCS}}^{R}$ [second and third diagram in Fig. 1(a)] contains only the retarded (advanced) photon GF $D_{R(A)}$, while the fluctuation term $\Gamma_{\text {fluct }}$ [fourth diagram in Fig. 1(a)] contains only $D_{K}$. Hence, the BCS term $\Gamma_{\mathrm{BCS}}$ is directly affected only by the dispersion and lifetime of the photons, but not by the their distribution. On the other hand, the fluctuation term $\Gamma_{\text {fluct }}$ knows about how photon modes are occupied. Correspondingly, while the BCS vertex contains the electron $G^{K}$ proportional to the Fermi-Dirac distribution, the fluctuation term only contains retarded electron GFs, i.e., it depends only on the electron dispersion and lifetime but not directly on their distribution.

Since the number of photons is not conserved and in the low-Kelvin regime there is essentially no thermal occupation of an optical cavity on average, the photons can be present only due to vacuum fluctuations (or if we include cavity loss by the corresponding noise, as we shall see later), which explains the nomenclature $\Gamma_{\text {fluct }}$.

Energy structure of the pairing.-Besides their complementarity in terms of the causal structure illustrated above, the fluctuation-induced pairing and the BCS pairing differ sharply in their energy structure. This can be understood by using the separation of energy scales between the on shell and off shell electrons, shown in Fig. 1(b). The on shell electrons have frequencies $\omega_{1} \sim \epsilon_{k_{F} \pm q_{0}}$ [see Eq. (3)], where $\epsilon_{k_{F} \pm q_{0}}$ is a small characteristic scale, i.e., $\epsilon_{k_{F} \pm q_{0}} \ll E_{F}$ as well as $\epsilon_{k_{F} \pm q_{0}} \ll \delta_{c}$. These low energy electrons, highlighted by the blue shell in Fig. 1(b), are the ones that eventually form the Cooper pairs, as signaled by a divergent pairing amplitude $\Gamma_{\text {on shell }}$ as a solution to the BS equation [Eq. (2)] for $T<T_{c}$. On the other hand, off shell electrons [highlighted by the green shell in Fig. 1(b)] have frequencies close to the photon resonance frequency, i.e., far away from the FS: $\omega_{1} \sim \delta_{c}$. The off shell electrons are not the ones actually building the pair, but can play a crucial role in intermediate scattering processes, as we shall see in the case when the fluctuation term is included.

$B C S$ pairing.-As illustrated in Fig. 1(c), here an adiabatic off shell photon with frequency $\omega_{1}-\omega \sim$ $\epsilon_{k_{F} \pm q_{0}} \ll \delta_{c}$ scatters an on shell electron (corresponding to the peak in $G^{K}$ around $\omega_{1} \sim \epsilon_{k_{F} \pm q_{0}}$ ) to a state which is still in the vicinity of the FS. This state is on shell since the transferred momentum $q_{0} \ll k_{F}$. In Eq. (3), $V^{(0)} D_{R(A)}$ in $\Gamma_{\mathrm{BCS}}$ can thus be substituted by a negative constant $\sim-g_{0}^{2} / 2 \delta_{c}$ quantifying the net attractive interaction, as in the standard BCS scenario involving phonons. This gives the following equation for $T_{c}^{\mathrm{BCS}}$ (see Appendix $\mathrm{C}$ in the Supplemental Material for a detailed derivation [24]):

$$
4 \tilde{g} \delta_{c} \frac{\tanh \left(\frac{\epsilon_{k_{F}+q_{0}}}{2 T_{c}^{\text {BCS }}}\right)}{\epsilon_{k_{F}+q_{0}}}=1 .
$$

Here, $\tilde{g}=g_{0}^{2} /\left(4 \pi \delta_{c}\right)^{2}$ is the dimensionless coupling. We observe that, differently from the standard phonon case, there are no integrals over loop momentum left due to the long-range nature of the photon-mediated interaction: $V^{(0)}(\vec{q}) \propto \delta_{\vec{q}, \pm q_{0} \hat{x}}$. For $T \gg \epsilon_{k_{F}+q_{0}}$, the linear vanishing $\tanh (x) \simeq x$ of the electron occupation is crucial in cutting off the $1 / \epsilon$ divergence at the FS $\left(\epsilon_{k_{F}+q_{0}} \propto q_{0}\right.$ is finite for finite $q_{0}$ but is the smallest scale), yielding $T_{c}^{\mathrm{BCS}} \sim 2 \tilde{g} \delta_{c}$.

Fluctuation-enhanced pairing.-On the other hand, photon fluctuations are concentrated around the cavity resonance frequency, which in the frame of the driving laser corresponds to $\delta_{c}$. Hence, in the fluctuation term, pairing is mediated by on shell nonadiabatic photons, with $\omega_{1}-\omega \sim \delta_{c}$ which scatter electrons off shell. The frequency dependence of the photon GF $D^{K}(\omega) \sim \delta\left(\omega-\delta_{c}\right)$ can never be neglected in Eq. (3). Because of the presence of $\Gamma_{\text {fluct }}$, the BS equation [Eq. (2)] is not diagonal in frequency, as it couples the electronic on shell and off shell sectors. As shown in Fig. 1(d), a further scattering process where a second nonadiabatic photon brings the electron back on shell closes the BS equation. The latter leads to the following equation for the critical temperature (see Appendix D in the Supplemental Material for a detailed derivation [24]):

$$
\frac{4 \tilde{g} \delta_{c}}{\epsilon_{k_{F}+q_{0}}} \tanh \left(\frac{\epsilon_{k_{F}+q_{0}}}{2 T_{c}^{\text {fluct }}}\right)+\frac{32}{3} \frac{\tilde{g}^{2} \delta_{c}^{2}}{\epsilon_{k_{F}+q_{0}}^{2}}\left(\operatorname{coth} \frac{\delta_{c}}{2 T_{c}^{\text {fluct }}}\right)^{2}=1 .
$$

Note that the BCS contribution [first term on the lhs of Eq. (6)] depends on the electron occupation, while the nonBCS-type contribution [second term on the lhs of Eq. (6)] depends on the photon occupation. Fig. 2(a) shows that the fluctuation-induced pairing significantly enhances superconductivity, leading to a critical temperature $T_{c}^{\text {fluct }}$ (dotted green line) increased by an order of magnitude with respect to the BCS prediction $T_{c}^{\mathrm{BCS}}$ (solid line with circles). This enhancement can be understood in the following way: for not too small laser detunings, the average thermal occupation of cavity photons is negligible: $\operatorname{coth}\left(\delta_{c} / 2 T\right) \sim 1$, so that only vacuum fluctuations remain (later we will discuss the impact of cavity loss and incoherent pump). This allows us to obtain the simple expression for the critical temperature $T_{c}^{\text {fluct }} \sim 2 \tilde{g} \delta_{c} /\left[1-32\left(\tilde{g} \delta_{c}\right)^{2} /\left(3 \epsilon_{k_{F}+q_{0}}^{2}\right)\right]$. For realistic 

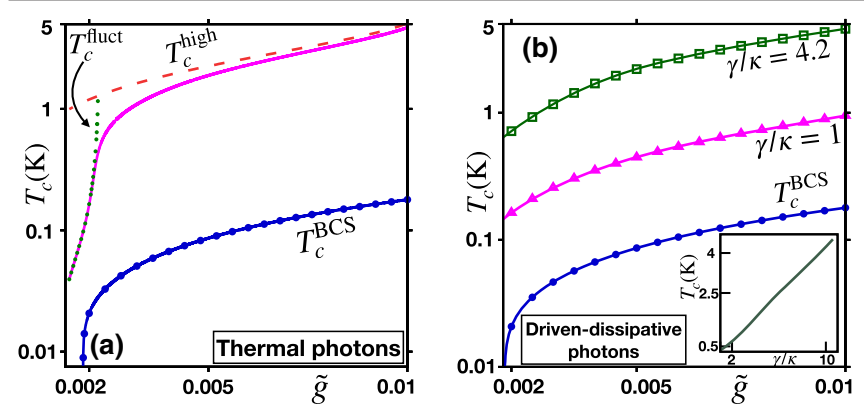

FIG. 2. Critical temperature $T_{c}$ vs dimensionless coupling strength $\tilde{g}$ in log plot. (a) For thermal photons, the blue solid line with circles corresponds to prediction (5), based on solely the BCS pairing [see Fig. 1(c)]. The solid magenta line corresponds to the prediction which also includes the fluctuation-induced pairing [see Fig. 1(d)], as well as a finite Fermi-liquid-type electron lifetime [see Eq. (7)]. The green dotted line shows the prediction for long-lived electrons from Eq. (6), while the red dashed line corresponds to the prediction of Eq. (8) for short-lived electrons. (b) For driven dissipative photons, solid line with circles show the BCS prediction which is almost unaffected by photon loss and incoherent pump. Solid lines with triangles show the fluctuation-assisted enhancement in the purely lossy case $\gamma=\kappa$ which is further amplified by incoherent pumping $\gamma>\kappa$ shown by solid line with squares. The inset (linear plot) shows $T_{c}$ increases almost linearly with $\gamma$. We choose $\delta_{c}=0.19 \mathrm{THz}$, $\epsilon_{k_{F}+q_{0}} / \delta_{c}=0.007, \kappa / \delta_{c}=0.01$, and for the inset $\tilde{g}=0.004$.

parameters of 2D materials (lanthanum aluminate-strontium titanate) coupled to a split-ring cavity [9,30,31], the ratio $\delta_{c} / \epsilon_{k_{F}+q_{0}} \sim 135$, and hence the fluctuation-induced term significantly reduces the denominator from 1 already at moderate coupling strengths $\tilde{g} \sim 0.002$, leading to a significant increase in $T_{c}$. We also note that the quantities appearing in the second term of Eq. (6) are squared due to the additional intermediate scattering to off shell states described above. The perturbative expansion in terms of such scattering processes is controlled as long as $\tilde{g}, \epsilon_{k_{F}+q_{0}} / \delta_{c} \ll 1$ (see Appendix D in the Supplemental Material [24]). Moreover, for the high-energy and lowmomentum photons involved in the non-BCS pairing, in Ref. [32] we show at the nonperturbative level that (i) particle-hole excitations only weakly affect the cavity-photon dynamics as well as the electron pairing and (ii) higher-order corrections to the electron-photon vertex are small. All these effects are thus safely neglected in our BS equation.

At the current level of description, the finite temperature cannot remove the $1 / \epsilon^{2}$ divergence at the FS arising from the non-BCS-type pairing. In order to remove this divergence, we need to take into account the finite lifetime of electrons. We consider here a Fermi-liquid scaling of the quasiparticle lifetime in two dimensions [33-35],

$$
\frac{1}{\tau_{e, \text { cou }}\left(T ; \epsilon_{k}\right)}=\frac{\pi}{8} \frac{\max \left(T, \epsilon_{k}\right)^{2}}{E_{F}} \log \left[\frac{E_{F}}{\max \left(T, \epsilon_{k}\right)}\right],
$$

induced by the screened Coulomb interaction between on shell electrons. This Fermi-liquid lifetime introduces also the leading temperature dependence of the fluctuationinduced pairing. This yields the critical temperature indicated by the solid (magenta) line in Fig. 2(a). For a finite cavity wave vector $q_{0}$ and at sufficiently low $T$, the quasiparticle energy $\epsilon_{k_{F}+q_{0}}$ dominates over the broadening $\tau_{e, \text { cou }}^{-1}\left(T ; \epsilon_{k}\right)$, so that $T_{c}$ closely follows the prediction $T_{c}^{\text {fluct }}$ for infinitely long-lived electrons. $T_{c}$ is raised further by increasing the coupling $\tilde{g}$, until the temperature becomes large enough for quasiparticle broadening to become appreciable, leading to the flattening of the critical-temperature curve as a function of $\tilde{g}$. A large enhancement induced by photon fluctuations still remains compared with the BCS prediction. In this regime, $\tau_{e \text {, cou }}^{-1}\left(T ; \epsilon_{k}\right) \gg \epsilon_{k_{F}+q_{0}}$, and the critical temperature is approximately determined by the following equation (see Appendix E in the Supplemental Material for a detailed derivation [24]):

$$
\frac{2 \tilde{g} \delta_{c}}{T_{c}^{\text {high }}}+\frac{32 \tilde{g}^{2} \delta_{c}^{2}}{\tau_{e, \text { cou }}^{-2}\left(T_{c}^{\text {high }}\right)}\left(\operatorname{coth} \frac{\delta_{c}}{2 T_{c}^{\text {high }}}\right)^{2}=1 .
$$

In the high $T$ limit, $T_{c}^{\text {high }}$ (dashed red line) shows good agreement with the full numerical answer. At this point, it is worthwhile to mention that $T_{c}^{\text {high }}$ is also valid in the limit of vanishing cavity wave vector $q_{0} \rightarrow 0$. Referring to the discussion made in the model section, we see that the fluctuation-assisted enhancement of superconductivity thus applies to both the split-ring-cavity geometry considered in Ref. [9] and to the planar microcavity geometry considered in Ref. [7].

Let us show more explicitly that the non-BCS-type pairing induced by fluctuations is appreciable only if the interactions are long ranged, i.e., when the bosonic mediator can transfer momenta which are concentrated in a narrow window. In order to obtain a simple estimate, we substitute the delta function in $V^{(0)}(\vec{q})$ with a box of fixed width. In this case, the factor $1 / \tau_{e, \text { cou }}^{-2}$ in the fluctuation term of Eq. (8) is replaced by

$$
-\frac{1}{2 W} \int_{-W}^{W} \frac{d \epsilon}{\left(\epsilon-\frac{\mathbf{i}}{\tau_{e, \text { cou }}}\right)\left(\epsilon-\frac{\mathbf{i}}{\tau_{e, \text { cou }}}\right)}=\frac{1}{W^{2}+\tau_{e, \text { cou }}^{-2}},
$$

where $W$ is the box width in units of energy. If the width $W$ exceeds the inverse electron lifetime $\tau_{e, \text { cou }}^{-1}$ at the Fermi surface, the fluctuation-induced enhancement of paring $\left(\propto \delta_{c}^{2} / W^{2}\right)$ will be cut off. This explains why the effect discussed in this work is not relevant for the standard phonon-mediated pairing. There the energy window $W$ is set by the Debye frequency, which is large compared with electronic scales. In this regime, the BCS term takes the known logarithmic form $T_{c}^{\mathrm{BCS}} \propto W \exp \left[-W /\left(\tilde{g} \delta_{c}\right)\right]$. 
Impact of photon loss and incoherent pump.-Photon loss out of the cavity mirrors is unavoidable and typically happens at an appreciable rate $\gamma_{\text {loss }}$. The resulting damping of photons is introduced in the retarded-advanced GFs $D_{R / A}$ in Eq. (4) by substituting $0^{+} \rightarrow \kappa$, while the corresponding noise is included through the Keldysh GF [36-38] as

$$
D_{K}(\vec{q}, \omega)=-\mathbf{i} \frac{\gamma}{\delta_{c}} \frac{\omega^{2}+\kappa^{2}+\delta_{c}^{2}}{\left(\omega^{2}-\kappa^{2}-\delta_{c}^{2}\right)^{2}+4 \kappa^{2} \omega^{2}} .
$$

Here the parameter $\gamma$ quantifies the noise level. If, apart from the coherent laser drive, the cavity is not further illuminated, then $\gamma=\kappa=\gamma_{\text {loss }} / 2$, i.e., the loss rate sets both the damping and the noise. We will also study the effect of an incoherent pump (as resulting from a broadband illumination) at rate $\gamma_{\text {pump }}<\gamma_{\text {loss }}$. In this case the net loss rate becomes $\kappa=\left(\gamma_{\text {loss }}-\gamma_{\text {pump }}\right) / 2$ while the total noise level $\gamma=\left(\gamma_{\text {loss }}+\gamma_{\text {pump }}\right) / 2>\kappa$. For any finite cavity-loss rate, inelastic electron-photon scattering further reduces the electron lifetime: $\tau_{e}^{-1}=\tau_{e, \text { cou }}^{-1}+\tau_{e, \text { cav }}^{-1}$, with $\tau_{e, \text { cav }}^{-1} \simeq$ $2 \tilde{g} \gamma /\left(1+\kappa^{2} / \delta_{c}^{2}\right)$ (see Appendix $\mathrm{F}$ in the Supplemental Material [24]). Assuming $\delta_{c} \gg \kappa$ and $\tau_{e}^{-1} \gg \epsilon_{k_{F}+q_{0}}$, the equation for the critical temperature takes the simple form (see Appendix F in the Supplemental Material [24] for a detailed derivation):

$\frac{2 \tilde{g} \delta_{c}}{T_{c}^{\text {noise }}}+4 \tilde{g}^{2} \frac{\gamma^{2}}{\kappa^{2}} \frac{\delta_{c}^{2}}{\tau_{e}^{-2}\left(T_{c}^{\text {noise }}\right)}\left[1-\frac{1}{1+\frac{\tau_{e}^{-1}\left(T_{c}^{\text {noise }}\right)}{2 \kappa}}\right]=1$.

This time we used $T_{c}^{\text {noise }}$ as opposed to $T_{c}^{\text {fluct }}$, since the presence of an on shell photon is not due to vacuum fluctuations but to loss-induced noise or, at finite pump rates, to a finite average occupation of the cavity mode. We see indeed that the thermal coth is replaced here by $\gamma / \kappa=1+2 n_{\mathrm{ph}}$, with $n_{\mathrm{ph}}$ being the average incoherent occupation of the cavity. When $\gamma_{\text {pump }}$ approaches $\gamma_{\text {loss }}$ our model needs to be extended to include pump saturation that prevents the divergence in the photon number. $T_{c}^{\text {noise }}$ is shown in Fig. 2(b). While the BCS prediction $T_{c}^{\mathrm{BCS}} \simeq$ $2 \tilde{g} \delta_{c} /\left(1+\kappa^{2} / \delta_{c}^{2}\right)$ [Fig. 2(b) with circles] remains essentially unaffected by loss (for $\delta_{c} \gg \kappa$ ) and does not depend on incoherent pumping, the non-BCS-type pairing still provides a strong enhancement of superconductivity [Fig. 2(b) with triangles], which is further amplified by a finite incoherent-pump rate [Fig. 2(b) with squares]. By comparing Eq. (11) with the closed-system expression, we see that for $\kappa \neq 0$ the critical temperature is reduced by the second term in the square bracket. Still, for $\kappa \tau_{e} \ll 1$ the reduction is negligible. Moreover, by increasing the incoherent-pump rate $\gamma$ we can further increase $T_{c}$ almost linearly, as shown in the inset of Fig. 2(b). Hence, with increasing $\gamma, \tau_{e, \text { cou }}^{-1}$ increases faster $\left(\propto T^{2} \log T\right)$ than the linearly increasing $\tau_{e, \text { cav }}^{-1}$. The Coulomb lifetime thus still serves as the dominant quasi-particle-damping process. For this reason, the photon-induced redistribution of quasiparticles, predicted to be appreciable in certain cavity setups [4] and observed by proper irradiation [39,40], can instead be neglected in our case. We can predict an increase in $T_{c}$ as long as the Fermi-liquid behavior (7) still holds, which sets $T_{c}$ in the range of the Fermi temperature $T_{F}(\sim 13 \mathrm{~K}$ for the materials considered in Ref. [9]).

Conclusions.-We have shown that cavity-mediated interactions between electrons induce a non-BSC-type of pairing mechanism triggered by nonadiabatic photon fluctuations, which can largely enhance the superconducting critical temperature $T_{c}$ under realistic conditions. The characteristic non-BCS dependence of $T_{c}$ on the tuneable light-matter coupling could be experimentally observed. Moreover, the presence of non-BCS pairing would be signalled by an increase of $T_{c}$ with the number incoherent photons in the cavity. These features could be also theoretically reproduced using quantum Monte Carlo for fermion-boson systems [41], proper extensions to longrange interactions of matrix product states [42] or density functional theory [43], as well as finite-frequency extensions of functional renormalization group technique [44].

We thank Bernhard Frank, Dieter Jaksch, Johannes Lang, Andrew Millis, Frank Schlawin, and Michael Sentef for useful discussions.

*ahana@pks.mpg.de piazza@pks.mpg.de

[1] G. L. Paravicini-Bagliani, F. Appugliese, E. Richter, F. Valmorra, J. Keller, M. Beck, N. Bartolo, C. Rössler, T. Ihn, K. Ensslin, C. Ciuti, G. Scalari, and J. Faist, Magnetotransport controlled by Landau polariton states, Nat. Phys. 15, 186 (2019).

[2] A. Thomas, E. Devaux, K. Nagarajan, T. Chervy, M. Seidel, D. Hagenmüller, S. Schütz, J. Schachenmayer, C. Genet, G. Pupillo, and T. W. Ebbesen, Exploring superconductivity under strong coupling with the vacuum electromagnetic field, arXiv:1911.01459.

[3] M. A. Sentef, M. Ruggenthaler, and A. Rubio, Cavity quantum-electrodynamical polaritonically enhanced electron-phonon coupling and its influence on superconductivity, Sci. Adv. 4, eaau6969 (2018).

[4] J. B. Curtis, Z. M. Raines, A. A. Allocca, M. Hafezi, and V. M. Galitski, Cavity Quantum Eliashberg Enhancement of Superconductivity, Phys. Rev. Lett. 122, 167002 (2019).

[5] G. Mazza and A. Georges, Superradiant Quantum Materials, Phys. Rev. Lett. 122, 017401 (2019).

[6] M. Kiffner, J. R. Coulthard, F. Schlawin, A. Ardavan, and D. Jaksch, Manipulating quantum materials with quantum light, Phys. Rev. B 99, 085116 (2019).

[7] F. Schlawin, A. Cavalleri, and D. Jaksch, Cavity-Mediated Electron-Photon Superconductivity, Phys. Rev. Lett. 122, 133602 (2019). 
[8] A. A. Allocca, Z. M. Raines, J. B. Curtis, and V. M. Galitski, Cavity superconductor-polaritons, Phys. Rev. B 99, 020504(R) (2019).

[9] H. Gao, F. Schlawin, M. Buzzi, A. Cavalleri, and D. Jaksch, Photoinduced Electron Pairing in a Driven Cavity, Phys. Rev. Lett. 125, 053602 (2020).

[10] Y. Ashida, A. Imamoglu, J. Faist, D. Jaksch, A. Cavalleri, and E. Demler, Quantum Electrodynamic Control of Matter: Cavity-Enhanced Ferroelectric Phase Transition, Phys. Rev. X 10, 041027 (2020).

[11] M. A. Sentef, J. Li, F. Künzel, and M. Eckstein, Quantum to classical crossover of Floquet engineering in correlated quantum systems, Phys. Rev. Research 2, 033033 (2020).

[12] F. Piazza and P. Strack, Umklapp Superradiance with a Collisionless Quantum Degenerate Fermi Gas, Phys. Rev. Lett. 112, 143003 (2014).

[13] J. Keeling, M. J. Bhaseen, and B. D. Simons, Fermionic Superradiance in a Transversely Pumped Optical Cavity, Phys. Rev. Lett. 112, 143002 (2014).

[14] Y. Chen, Z. Yu, and H. Zhai, Superradiance of Degenerate Fermi Gases in a Cavity, Phys. Rev. Lett. 112, 143004 (2014).

[15] F. Piazza and P. Strack, Quantum kinetics of ultracold fermions coupled to an optical resonator, Phys. Rev. A 90, 043823 (2014).

[16] J.-S. Pan, X.-J. Liu, W. Zhang, W. Yi, and G.-C. Guo, Topological Superradiant States in a Degenerate Fermi Gas, Phys. Rev. Lett. 115, 045303 (2015).

[17] W. Zheng and N. R. Cooper, Superradiance Induced Particle Flow via Dynamical Gauge Coupling, Phys. Rev. Lett. 117, 175302 (2016).

[18] C. Kollath, A. Sheikhan, S. Wolff, and F. Brennecke, Ultracold Fermions in a Cavity-Induced Artificial Magnetic Field, Phys. Rev. Lett. 116, 060401 (2016).

[19] F. Mivehvar, H. Ritsch, and F. Piazza, Superradiant Topological Peierls Insulator inside an Optical Cavity, Phys. Rev. Lett. 118, 073602 (2017).

[20] E. Colella, R. Citro, M. Barsanti, D. Rossini, and M.-L. Chiofalo, Quantum phases of spinful Fermi gases in optical cavities, Phys. Rev. B 97, 134502 (2018).

[21] F. Mivehvar, H. Ritsch, and F. Piazza, Cavity-QuantumElectrodynamical Toolbox for Quantum Magnetism, Phys. Rev. Lett. 122, 113603 (2019).

[22] F. Schlawin and D. Jaksch, Cavity-Mediated Unconventional Pairing in Ultracold Fermionic Atoms, Phys. Rev. Lett. 123, 133601 (2019).

[23] A. A. Abrikosov, I. Dzyaloshinskii, L. P. Gorkov, and R. A. Silverman, Methods of Quantum Field Theory in Statistical Physics (Dover, New York, NY, 1975).

[24] See Supplemental Material at http://link.aps.org/ supplemental/10.1103/PhysRevLett.127.177002 for the derivation of the effective action using the Schwinger-Keldysh field theoretic technique, the solution of the resultant BetheSalpeter equation for the vertex-function to find critical temperature including both the standard BCS and the new non-BCS mechanisms, a discussion on the effect of finite lifetime of the electrons and the role cavity-losses and incoherent pumping, which includes Refs. [25-27].

[25] J. W. Negele and H. Orland, Quantum Many Body Systems (Oxford University Press on Demand, New York, 2002).
[26] A. Altland and B. D. Simons, Condensed Matter Field Theory (Cambridge University Press, Cambridge, England, 2010).

[27] D. Pines and P. Nozières, The Theory of Quantum Liquids Normal Fermi Liquids (CRC Press, Taylor and Francis Group, Florida, 2018), pp. 1-180.

[28] R. D. Mattuck, Methods of Quantum Field Theory in Statistical Physics (Dover Publications, INC., New York, 1992).

[29] A. Kamenev, Field Theory of Non-Equilibrium Systems (Cambridge University Press, Cambridge, England, 2011).

[30] T. Sakudo and H. Unoki, Dielectric Properties of $\mathrm{SrTiO}_{3}$ at Low Temperatures, Phys. Rev. Lett. 26, 851 (1971).

[31] X. Lin, Z. Zhu, B. Fauqué, and K. Behnia, Fermi Surface of the Most Dilute Superconductor, Phys. Rev. X 3, 021002 (2013).

[32] A. Chakraborty and F. Piazza (to be published).

[33] G. F. Giuliani and J. J. Quinn, Lifetime of a quasiparticle in a two-dimensional electron gas, Phys. Rev. B 26, 4421 (1982).

[34] L. Zheng and S. Das Sarma, Coulomb scattering lifetime of a two-dimensional electron gas, Phys. Rev. B 53, 9964 (1996).

[35] T. Jungwirth and A.H. MacDonald, Electron-electron interactions and two-dimensional-two-dimensional tunneling, Phys. Rev. B 53, 7403 (1996).

[36] E. G. D. Torre, S. Diehl, M. D. Lukin, S. Sachdev, and P. Strack, Keldysh approach for nonequilibrium phase transitions in quantum optics: Beyond the Dicke model in optical cavities, Phys. Rev. A 87, 023831 (2013).

[37] L. M. Sieberer, M. Buchhold, and S. Diehl, Keldysh field theory for driven open quantum systems, Rep. Prog. Phys. 79, 096001 (2016).

[38] J. Lang, D. E. Chang, and F. Piazza, Nonequilibrium diagrammatic approach to strongly interacting photons, Phys. Rev. A 102, 033720 (2020).

[39] T. Klapwijk and P. de Visser, The discovery, disappearance and re-emergence of radiation-stimulated superconductivity, Ann. Phys. (Amsterdam) 417, 168104 (2020).

[40] K. S. Tikhonov, A. V. Semenov, I. A. Devyatov, and M. A. Skvortsov, Microwave response of a superconductor beyond the Eliashberg theory, Ann. Phys. (Amsterdam) 417, 168101 (2020).

[41] S. Beyl, F. Goth, and F. F. Assaad, Revisiting the hybrid quantum Monte Carlo method for Hubbard and electronphonon models, Phys. Rev. B 97, 085144 (2018).

[42] C.-M. Halati, A. Sheikhan, and C. Kollath, Theoretical methods to treat a single dissipative bosonic mode coupled globally to an interacting many-body system, Phys. Rev. Research 2, 043255 (2020).

[43] P. de Silva, T. Zhu, and T. Van Voorhis, Long-range interactions from the many-pair expansion: A different avenue to dispersion in DFT, J. Chem. Phys. 146, 024111 (2017).

[44] C. Karrasch, R. Hedden, R. Peters, T. Pruschke, K. Schönhammer, and V. Meden, A finite-frequency functional renormalization group approach to the single impurity Anderson model, J. Phys. Condens. Matter 20, 345205 (2008). 